\title{
Locally advanced adenocarcinoma of the cervix on uterus didelphys: a case report
}

\author{
Abel Cordoba, MD, Alexandre Escande, MD, Pauline Comte, MD, Ingrid Fumagalli, MD, Lucie Bresson, MD, \\ Ndaye Mubiayi, MD, Eric Lartigau, MD, PhD \\ Department of Radiotherapy, Oscar Lambret Comprehensive Cancer Center, Lille, France
}

\begin{abstract}
In November 2013, a woman with Herlyn-Werner-Wunderlich (HWW) syndrome was diagnosed with a locally advanced left cervical adenocarcinoma. The patient's malformation consisted of two uteri with two cervixes, a obstructed vagina, and a left renal agenesis. Classification FIGO: stage IIIa because of infiltration of the inferior third of the vagina wall. Locoregional management comprised an infrarenal lateral aortic lymphadenectomy followed by concomitant radiochemotherapy to the pelvic (inguinal, pelvic, and infrarenal para aortic nodes) volumes. A total of 50.4 Gy were delivered (1.8 Gy/fraction/day) to the node (inguinal, pelvic, and aortic infrarenal) and pelvic volume; a concomitant boost to the primary cervical tumor and macroscopic nodes to $59.92 \mathrm{~Gy}$ (2.14 Gy/fraction/day) was performed. 20 Gy were delivered with intracavitary brachytherapy boost with mold technique and a pulsed-dose-rate technique due to the rarity of this uterine malformation. After 30 months of follow-up, there was no evidence of locoregional or distant recurrence.

Key words: cervix cancer, cervical cancer, pulsed-dose-rate, uterus didelphys.

\section{Purpose}

Cervical cancer is one of the most common cancers in women worldwide [1]. For locally advanced cervical cancer, concomitant radio-chemotherapy (RCT) followed by 3D intracavitary brachytherapy (IBT) is the standard of care of treatment [2,3]. Herlyn-Werner-Wunderlich (HWW) syndrome is a rare anomaly $[4,5]$ that represents a type of Müllerian duct anomalies (MDA), associated with mesonephric duct anomalies [6]. The prevalence is $0.5-1.0 \%$ of women. Herlyn-Werner-Wunderlich syndrome is a rare form of uterovaginal duplication with three characteristic anomalies, namely, didelphys uterus, unilateral obstructed hemivagina, and ipsilateral renal agenesis. However, less than $10 \%$ of this patients population, presents the entire triad. Herlyn-Werner-Wunderlich syndrome results from an embryologic aberration occurring during the eighth week of gestation that simultaneously involves the metanephric ducts as well as the paramesonephric or Müllerian ducts. There are only data from isolated case reports published for this type of disease $[5,7,8,9,10]$. We performed a concomitant RCT for a locally advanced adenocarcinoma cervical cancer, followed by a computed tomography (CT)-guided IBT for a patient with HWW syndrome.

\section{Clinical case}

The patient was a 37-year-old premenopausal and multiparous woman who presented with menorrhagia and
3 months of intermenstrual bleeding. She had class I HWW syndrome with class III Müllerian duct anomalies (two uteri and two cervixes), associated with left renal agenesis. The left uterus was initially blinded. Herlyn-Werner-Wunderlich was diagnosed in 2001 with left cervix hematocolpos; marsupialization was the surgical treatment. She had 2 pregnancies in her right uterus and had a C-section (as well as two voluntary interruptions and three miscarriages).

Clinical examination found a bulky cervical tumor with a proximal left parametrial invasion and an inferior vagina fornix extension. The biopsy result was a left cervix adenocarcinoma.

\section{Staging}

Physical examination showed an exophytic lesion in the upper third of the vagina in its left side. Examination with a speculum also showed a bleeding lesion in the left side of the upper third of the vagina.

A pelvic magnetic resonance imaging (MRI) found a $34 \times 57 \times 42 \mathrm{~mm}$ cervical mass arising from the left cervix with an inferior left posterior vagina wall and left proximal parametrial extension (Figure 1). According to the FIGO (Federation Internationale de Gynecologie et d'Obstetrique) classification, the tumor was stage IIIa. The estimated tumor volume was $36.3 \mathrm{~cm}^{3}$. A positron emission tomography-computed tomography (PET-CT) exam confirmed the primary cervical tumor with a hyper- 


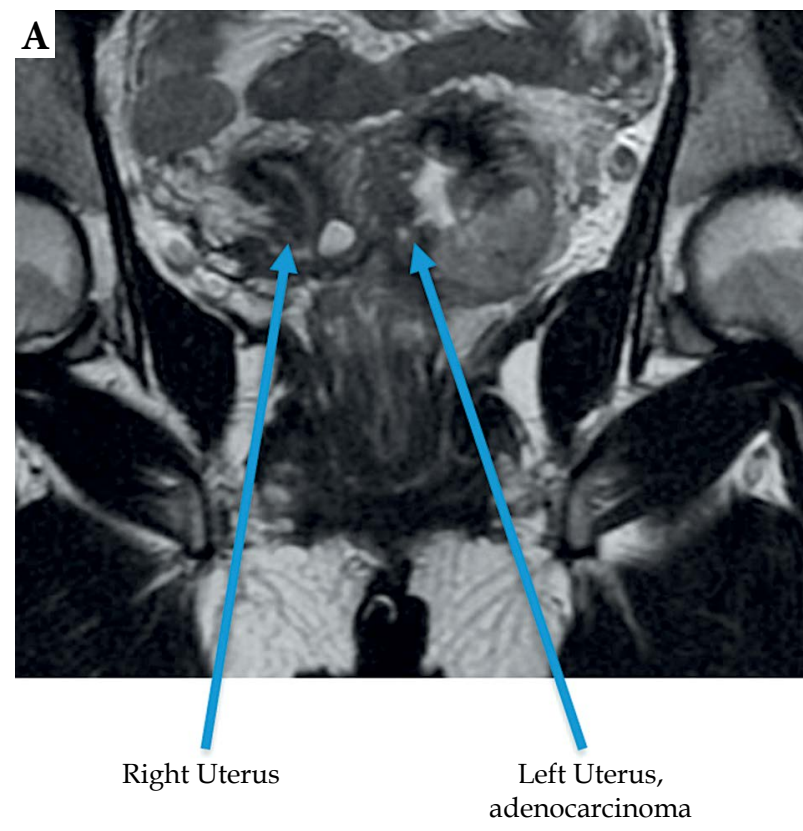

Fig. 1. Pelvis magnetic resonance imaging scan before treatment: A) sagittal view, B) coronal view

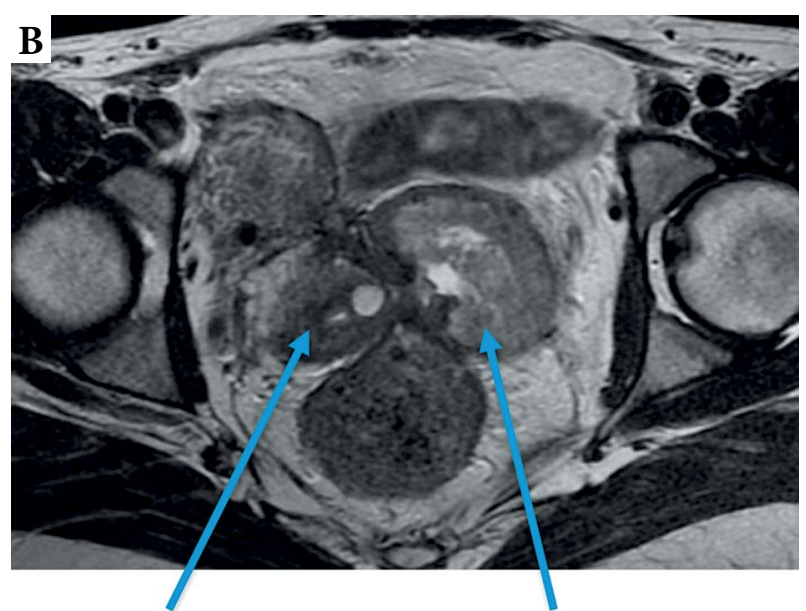

Right Cervix

Left Cervix, adenocarcinoma metabolic internal left iliac node (SUV max: $16.3 \mathrm{~g} / \mathrm{m}$ ) (Figure 2). Due to the existence of only a right kidney, a laparoscopic inframesenteric lymphadenectomy was performed that found two extracapsular well-differentiated adenocarcinoma nodes and no ovary infiltration. No pelvic nodal examination was performed due to anatomical difficulties.

During the surgery, a ureteral endoprosthesis was inserted into the right urethra in order to preserve the right kidney during RCT.

\section{Treatment}

Radio-chemotherapy: We treated two clinical target volumes (CTV) - CTV 1 included the center-pelvic gross tumor volume (GTV), as well as a vaginal safety margin of $4 \mathrm{~cm}$ below the tumor, pelvis with inguinal nodes due to inferior vaginal wall invasion, and para-aortic nodes due to positivity of lombo-aortic node dissection, the entire uterus, both parametria, and the involved portion of sacro-uterine ligaments. The GTV included the centro-pelvic gross tumor and pelvic-paraaortic nodes. CTV 2 included the GTV tumor and pelvic-paraaortic nodes plus $4 \mathrm{~mm}$ of margin [11]. Planning target volume (PTV) 1 and PTV 2 were defined as CTV 1 and CTV 2 expansions of $4 \mathrm{~mm}$, consistent with our previous published protocol [12,13]. We delivered 50.4 (1.8 Gy/fx/d) in the PTV 1, and 59.92 Gy $(2.14 \mathrm{~Gy} / \mathrm{fx} / \mathrm{d})$ in the PTV 2 simultaneously in 28 fractions (5 fractions a week). The maximum tolerated doses to the organs at risk (OAR) were as follows: for the small bowel bag, 50 Gy was the maximal tolerated dose, and $\mathrm{V}_{45}$ and $\mathrm{V}_{40}$ had to be $<50 \mathrm{cc}$ and $200 \mathrm{cc}$, respectively. For the bladder, rectum, and sigmoid structures, $60 \mathrm{~Gy}$ was the maximal dose, and $\mathrm{V}_{45}$ and $\mathrm{V}_{40}$ were $<20 \%$ and $<50 \%$, respectively. Treatment was performed using helical tomotherapy (Accuray Incorporated, Sunnyvale, CA, USA) with daily megavoltage computed tomography
(MVCT) technique with $6 \mathrm{MV}$ photons. During the treatment, which ran from November 14, 2013, to December 26, 2013 , the patient received 5 weekly cisplatin chemotherapy cycles $\left(40 \mathrm{mg} / \mathrm{m}^{2}\right)$.

\section{Intracavitary brachytherapy}

On January 2, 2014, IBT was performed under general anesthesia after a careful clinical examination that showed no residual tumor. We used the vaginal mold technique because of the patient's uncommon anatomy (Figure 3). Computer tomography was done to perform planning treatment (Figure 4). The high-risk clinical volume (HR-CTV) was contoured per GEC-ESTRO (Groupe Européen de Curiethérapie [GEC] and European Society for Radiotherapy \& Oncology [ESTRO]) guidelines. Treatment was performed via pulsed-dose-rate (PDR) technique with 40 pulses of $0.5 \mathrm{~Gy} /$ hour, for a total dose of $20 \mathrm{~Gy}$.

Organs at risk (OARs) parameters were as follows: rectum $\mathrm{D}_{2 \mathrm{cc}} \mathrm{EQD}_{2}<70 \mathrm{~Gy}$, sigmoid $\mathrm{D}_{2 \mathrm{cc}} \mathrm{EQD}_{2}<70 \mathrm{~Gy}$, and bladder $\mathrm{D}_{2 \mathrm{cc}} \mathrm{EQD}_{2}<85 \mathrm{~Gy}$. To calculate the dose from combined external beam radiation therapy (EBRT) and brachytherapy, it was assumed that the entire HRCTV of the IBT and the OARs received $100 \%$ of the prescribed external beam radiation dose. Equivalent biological doses to normal fractionation values were calculated as $\alpha / \beta=10$ for tumors and $\alpha / \beta=3$ for OARs, with a repair halftime of $1.5 \mathrm{~h}$.

\section{Results}

\section{External beam radiation therapy dosimetry}

For PTV 1, $\mathrm{D}_{98 \%}$ and $\mathrm{D}_{2 \%}$ was $47.06 \mathrm{~Gy}$ and $60.4 \mathrm{~Gy}$, respectively. For PTV 2, $D_{98 \%}$ was 57.27 Gy and $D_{2 \%}$ was $60.8 \mathrm{~Gy}$, respectively. The doses that OARs received are presented in Table 1 . Acute complications were abdominal pain grade II that was treated with oxycodone 

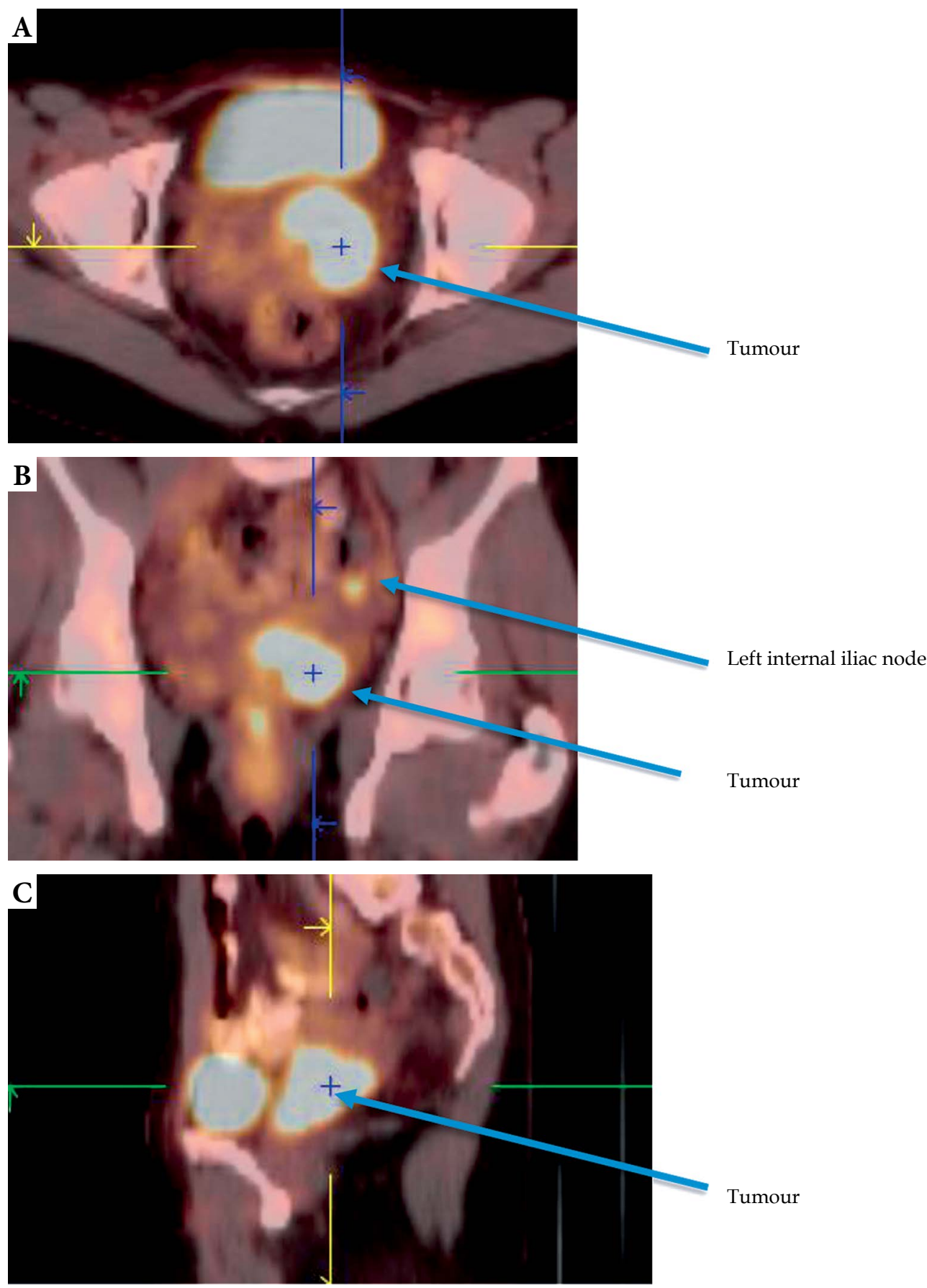

Fig. 2. Positron emission tomography scans before treatment: A) axial, tumor view, B) coronal, tumor and left internal iliac node view, C) sagittal tumor view

(NCI-CTCAE 4.03 scale), and nausea grade II that was treated with ondansetron. At the end of the treatment, a new clinical examination and a new MRI were performed with complete tumor response (Figure 5).

\section{Brachytherapy dosimetry}

Dose volume histogram $(\mathrm{DVH})$ for $\mathrm{HR}-\mathrm{CTV}, \mathrm{V}_{40 \mathrm{~Gy}}=$ $34.65 \%, \mathrm{D}_{95 \%}=13.6 \mathrm{~Gy}$, and $\mathrm{D}_{90 \%}=16.4 \mathrm{~Gy}$.

Treatment was delivered with a PDR of ${ }^{192} \mathrm{Ir}$ afterloading system. No acute toxicity during IBT treatment was observed.

\section{Total dosimetry (radio-chemotherapy + intracavitary brachytherapy)}

$\mathrm{D}_{90}$ to HR-CTV was 76.5 Gy $\left(\mathrm{EQD}_{2} \mathrm{~Gy}\right)$ with an $\alpha / \beta$ ratio of 10 . Total treatment time was 52 days.

\section{Follow-up}

Follow-up of this patient was made by clinical examination, image by pelvic MRI and because histology was adenocarcinoma, CA-125 marker level was performed every 4 months. At 24 months of follow-up, 

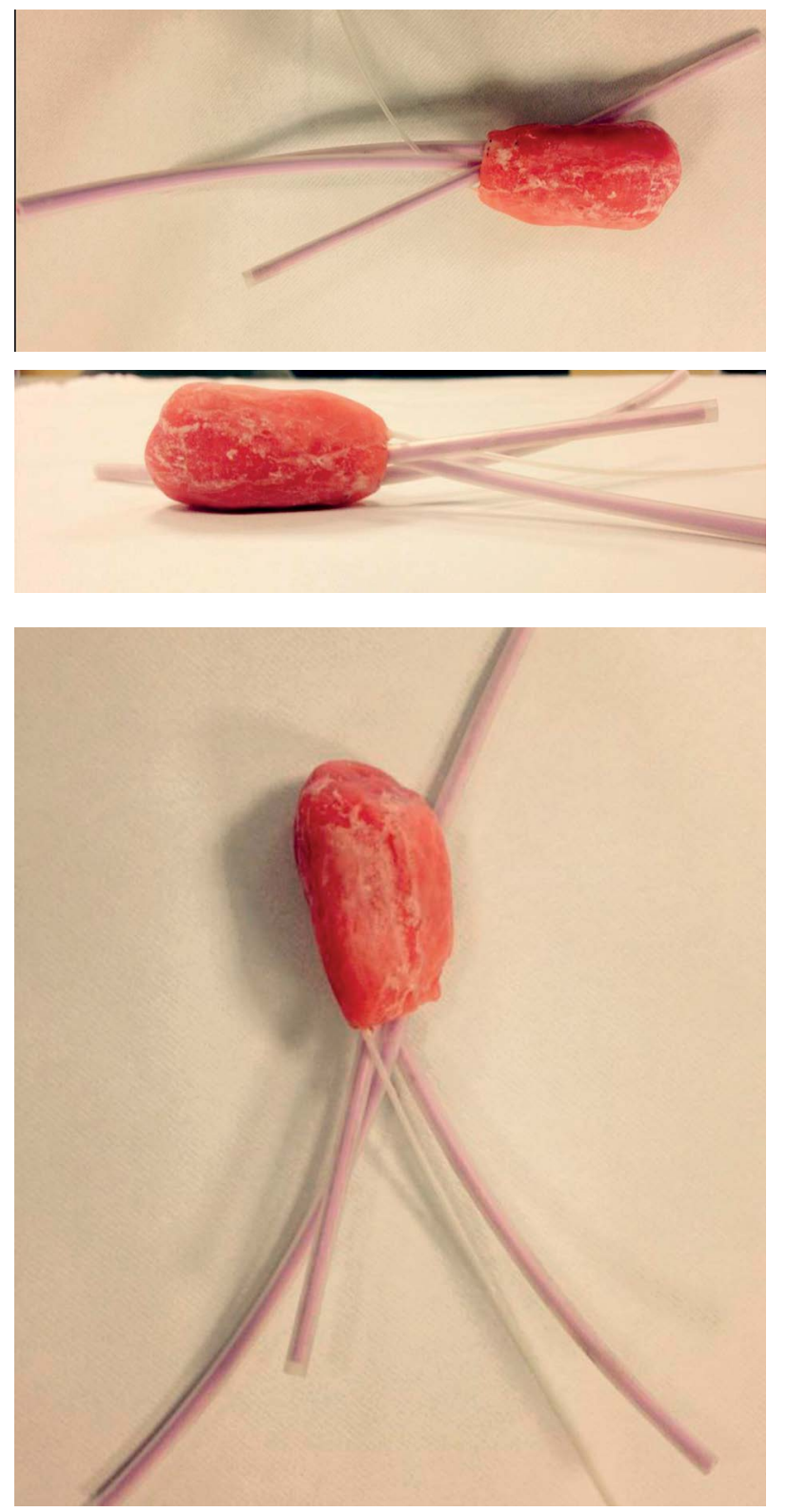

Fig. 3. Vaginal mold

there were no clinical or radiological (with an MRI performed every 6 months) signs of local or locoregional relapse.

Late complication was evaluated according to $\mathrm{NCI}$ CTCAE 4.03 (National Cancer Institute CTCAE - Common Terminology Criteria for Adverse Events) with no complications but minor sexual trouble with vaginal dryness grade I. A rectoscopy in January 2015 found a rectitis without symptom.

\section{Discussion}

Association between RCT and IBT is the standard treatment of locally advanced cervix carcinoma. Intracavitary brachytherapy is essential for cervical tumors due to its high gradient dose sparing the bladder, rectum, and small bowel.
Herlyn-Werner-Wunderlich syndrome is a rare variant of Müllerian duct anomalies consisting of uterine didelphys, obstructed hemivagina, and ipsilateral renal agenesis. Failure of complete lateral fusion of the paramesonephric ducts results in uterus didelphys, and a lack of development of the metanephric duct resulting in ipsilateral renal agenesis.

Most of women are diagnosed at the age of menarche; the preferred surgical approach for patients with HWW syndrome is full excision and marsupialization of the vaginal septum, preserving reproductive capacity [14]. Due to patient's special anatomy and locally advanced tumor close to the left pelvic wall, we preferred to elaborate a plan of treatment advocating external beam radiotherapy; in this sense, we delivered 50.4 Gy in 28 daily sessions of 1.8 Gy to the pelvic volume with a simultaneous integrated boost to the primary tumor of 59.92 (28 daily sessions of $2.14 \mathrm{~Gy}$ ).

At our institution, an MRI of 45-50 Gy to the pelvic volume is always performed to assess tumor evolution. In this clinical report, we observe that there was no residual tumor at IBT time (a pelvic MRI was performed 8 days before the IBT program date when 53.50 Gy had been delivered to PTV 2).

Intracavitary brachytherapy must be reached to obtain the best rate of local control $[15,16]$. But the anatomical anomalies made a good quality of implant difficult. Less than $1 \%$ of patients present such anomalies of the reproductive tract, so the incidence of cancer in this case is very rare. In our case report, the mold technique was used because no commercialized applicators could have been used in the special anatomy of this patient. Due to the lateralized character of this entity, we decided to insert only into the left uterine cavity, and the mold was designed to cover the left vaginal wall, left cervical area, and tumor extension to the left uterus.

The PDR technique was used to reduce the number of applications and thus avoid differences in applicator positioning (classically, 3 or 4 applications in high-doserate [HDR] brachytherapy) due to the special anatomy of the patient. Three-dimensional planning was performed thanks to a CT, which helped delineate the target volume and for manual optimization.

Such cases are described quite rare. Three clinical reports have been published till date about this clinical setting.

Lee et al. [8] reported a patient case with a didelphys uterus, class III Müllerian duct anomaly who presented a FIGO stage IIA1 tumor in the left cervix. They performed a whole pelvic EBRT to $45 \mathrm{~Gy}$ in 25 fractions, followed by IBT treatment performed placing a tandem into each cervical and uterine cavity. The dose was referred to adapted point $\mathrm{A}, 2 \mathrm{~cm}$ lateral and superior to each cervical structure. Two fractions of 6 and 6.5 Gy HDR modality were delivered; after nearly 4 years of follow-up, there was no local recurrence.

Loo and Locks [7] reported a case of a patient with a bicornuate bicollis uterus (class IV Müllerian duct anomaly) who presented a FIGO stage IIA2 cervical cancer. They performed a 50 Gy whole pelvis EBRT with concurrent chemotherapy (five cycles of concurrent cisplatin $40 \mathrm{mg} / \mathrm{m}^{2}$ ), followed by IBT. Also, 2 points A were de- 

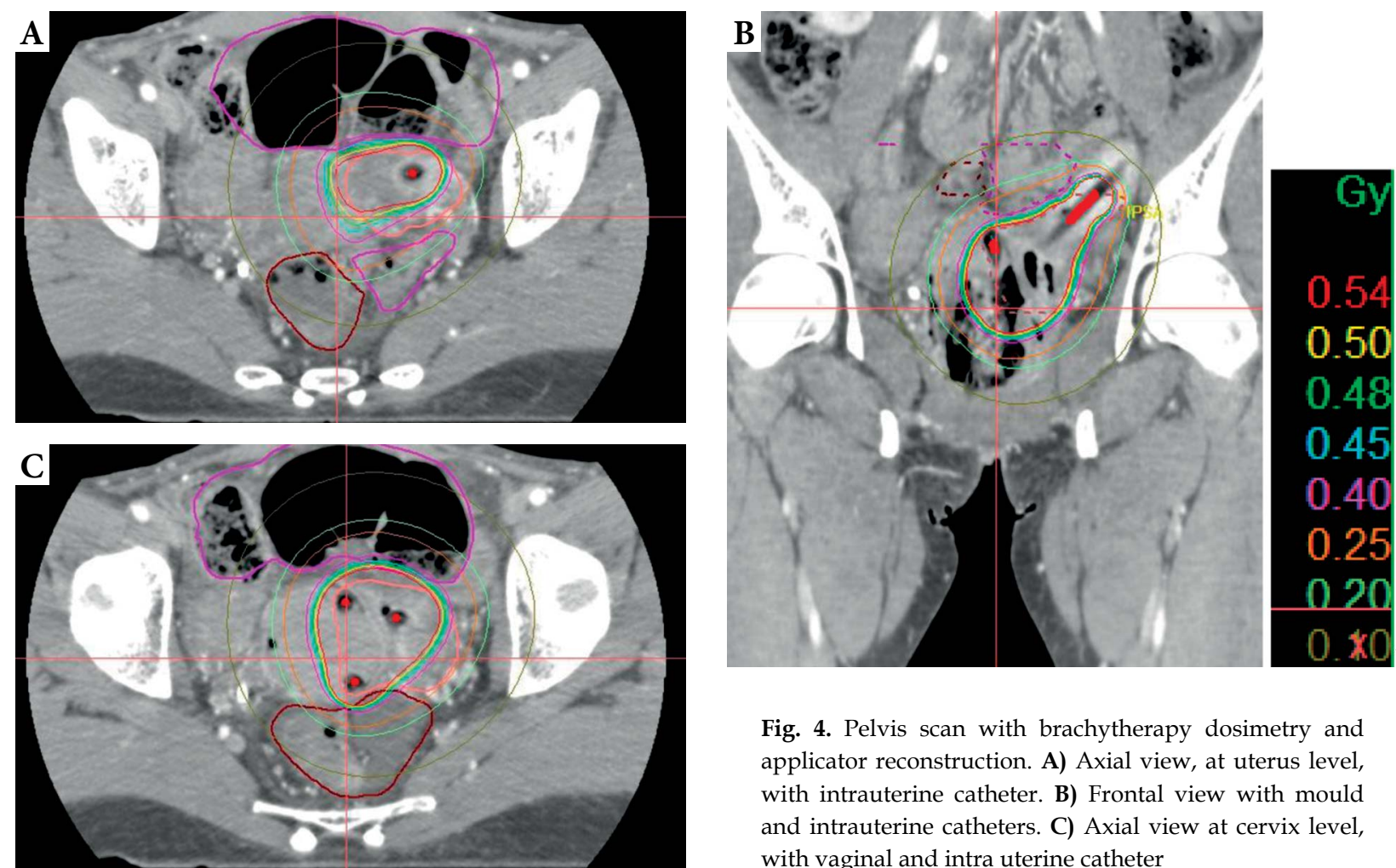

Fig. 4. Pelvis scan with brachytherapy dosimetry and applicator reconstruction. A) Axial view, at uterus level, with intrauterine catheter. B) Frontal view with mould and intrauterine catheters. C) Axial view at cervix level, with vaginal and intra uterine catheter

fined, considering special anatomy and 2 fractions of $18 \mathrm{~Gy}$ in a low-dose-rate (LDR) were delivered. After 2 years of follow-up, there was no evidence of local recurrence.

Platta et al. [5] reported a case of a patient with a septate uterus (Class V Müllerian duct anomaly) who presented a FIGO stage IIB tumor. They performed a whole pelvic EBRT to 45 Gy in 25 fractions with concurrent chemotherapy, followed by a cervical IBT using a Rotte " $Y$ " tandem and 2 ovoids. Five fractions of 5.5 Gy each were delivered with CT-based planning. No local recurrence had been achieved after 20 months of follow-up.

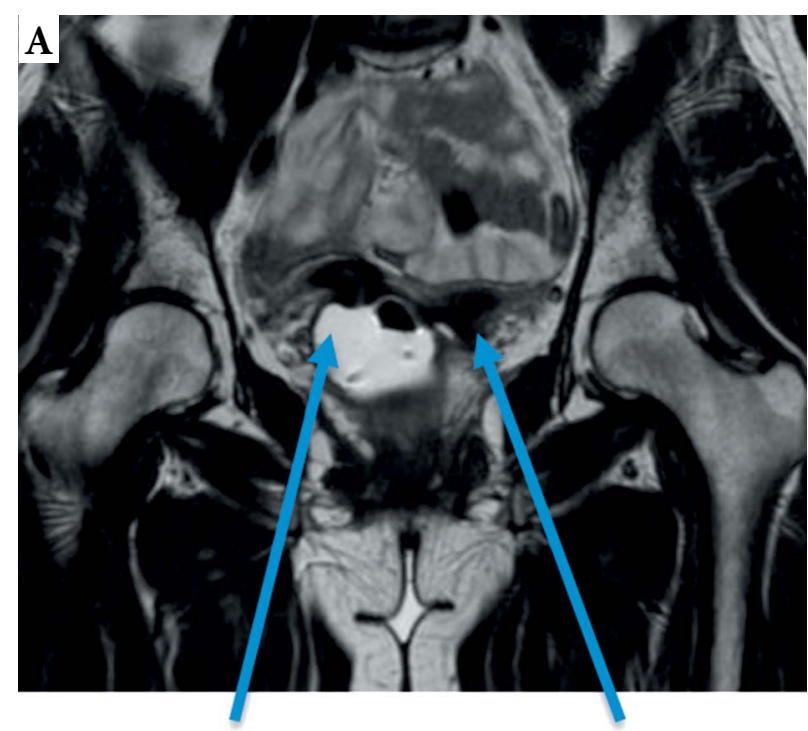

Table 1. Dose volume histograms for organs at risk equivalent dose at 2 Gy (external beam radiation therapy + brachytherapy)

\begin{tabular}{lc} 
Organs at risk & $\mathrm{D}_{2 \mathrm{cc}}$ \\
\hline Bladder & $62.4 \mathrm{~Gy}$ \\
\hline Rectum & $66.75 \mathrm{~Gy}$ \\
\hline Sigmoid & $58.4 \mathrm{~Gy}$ \\
\hline Small intestine & $57.5 \mathrm{~Gy}$
\end{tabular}

$D_{2 c c}$-minimum dose to the most exposed $2 \mathrm{~cm}^{3}$

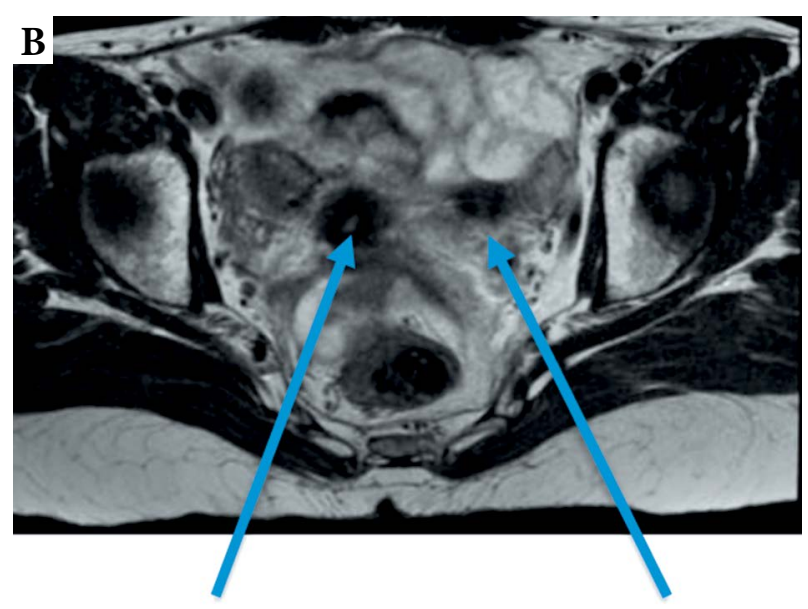

Right normal Cervix

Left normal Cervix

Right Uterus

Left normal Uterus

Fig. 5. Pelvis magnetic resonance imaging scan post-external beam radiation therapy: A) frontal view, B) axial view 
Integration of image advances in IBT planning treatment as MRI has to be adopted in daily practice to deliver higher doses to 3D known volumes, sparing the rectal area and the urinary bladder [17].

The treatment of parametrial invasion is controversial according to literature. There are several publications that show higher local control using interstitial needles such as Vienna and Utrecht applicators (Nucletron, an Elekta company, Elekta AB, Stockholm, Sweden) [18]. We choose to treat parametrial invasion in concomitant boost associated with a primary cervical tumor related to IBT complement. Regarding the choice of the brachytherapy technique used and knowing that there are no differences between PDR and HDR, PDR was chosen to ensure the same implant throughout the IBT treatment and to reduce possible errors due to multiple applications $[19,20]$.

\section{Conclusions}

In our case report, conventional external radiation therapy and concomitant chemotherapy with simultaneous concomitant boost to primary tumor and pathological pelvic lymph nodes followed by IBT was feasible with no evidence of disease at 24 months of follow-up and no major digestive, urinary, or sexual toxicities.

\section{Disclosure}

Authors report no conflict of interest.

\section{References}

1. Torre LA, Bray F, Siegel RL et al. Global cancer statistics, 2012. CA Cancer J Clin 2015; 65: 87-108.

2. Chemoradiotherapy for Cervical Cancer Meta-Analysis Collaboration. Reducing uncertainties about the effects of chemoradiotherapy for cervical cancer: a systematic review and meta-analysis of individual patient data from 18 randomized trials. J Clin Oncol 2008; 26: 5802-5812.

3. Haie-Meder C, Pötter R, Van Limbergen E et al. Recommendations from Gynaecological (GYN) GEC-ESTRO Working Group (I): concepts and terms in 3D image based 3D treatment planning in cervix cancer brachytherapy with emphasis on MRI assessment of GTV and CTV. Radiother Oncol 2005; 74: 235-245.

4. The American Fertility Society classifications of adnexal adhesions, distal tubal occlusion, tubal occlusion secondary to tubal ligation, tubal pregnancies, müllerian anomalies and intrauterine adhesions. Fertil Steril 1988; 49: 944-955.

5. Platta CS, Wallace C, Gondi V et al. Cervical brachytherapy technique for locally advanced carcinoma of the cervix in a patient with septate uterus. J Contemp Brachytherapy 2014; 6: 76-81.

6. Del Vescovo R, Battisti S, Di Paola V et al. Herlyn-WernerWunderlich syndrome: MRI findings, radiological guide (two cases and literature review), and differential diagnosis. BMC Med Imaging 2012; 12: 4.

7. Loo HW, Locks SM. Squamous cell carcinoma of the cervix: report of an unusual case of bicornuate bicollis uterus treated with bilateral intracavity brachytherapy. Br J Radiol 2010; 83: e143-146.

8. Lee CD, Churn M, Haddad N et al. Bilateral radical radiotherapy in a patient with uterus didelphys. Br J Radiol 2000; 73: 553-556.
9. Sharma R, Dey AK, Mittal K et al. Use of imaging in diagnosis of Herlyn-Werner-Wunderlich syndrome, a case report. J Obstet Gynaecol 2016; 36: 873-875.

10. Mehra S, Chamaria K, Garga UC et al. Imaging Diagnosis of Herlyn-Werner-Wunderlich Syndrome - An Extremely Rare Urogenital Anomaly. J Clin Diagn Res 2015; 9: TD06-8.

11. Lim K, Small W, Portelance L et al. Consensus guidelines for delineation of clinical target volume for intensity-modulated pelvic radiotherapy for the definitive treatment of cervix cancer. Int J Radiat Oncol Biol Phys 2011; 79: 348-355.

12. Mouttet-Audouard R, Lacornerie T, Tresch E et al. What is the normal tissues morbidity following Helical Intensity Modulated Radiation Treatment for cervical cancer? Radiother Oncol 2015; 115: 386-391.

13. Le Tinier F, Reynaert N, Castelain B et al. Is adaptive intensity-modulated radiotherapy for uterine cervix carcinoma necessary? Cancer Radiother 2012; 16: 681-687 [Article in French].

14. Gholoum S, Puligandla PS, Hui T et al. Management and outcome of patients with combined vaginal septum, bifid uterus, and ipsilateral renal agenesis (Herlyn-Werner-Wunderlich syndrome). J Pediatr Surg 2006; 41: 987-992.

15. Gill BS, Lin JF, Krivak TC et al. National Cancer Data Base analysis of radiation therapy consolidation modality for cervical cancer: the impact of new technological advancements. Int J Radiat Oncol Biol Phys 2014; 90: 1083-1090.

16. Han K, Milosevic M, Fyles A et al. Trends in the utilization of brachytherapy in cervical cancer in the United States. Int J Radiat Oncol Biol Phys 2013; 87: 111-119.

17. Dimopoulos JCA, Pötter R, Lang $S$ et al. Dose-effect relationship for local control of cervical cancer by magnetic resonance image-guided brachytherapy. Radiother Oncol 2009; 93: 311-315.

18. Bailleux C, Falk AT, Chand-Fouche ME et al. Concomitant cervical and transperineal parametrial high-dose-rate brachytherapy boost for locally advanced cervical cancer. J Contemp Brachytherapy 2016; 8: 23-31.

19. Haie-Meder C, Chargari C, Rey A et al. MRI-based low doserate brachytherapy experience in locally advanced cervical cancer patients initially treated by concomitant chemoradiotherapy. Radiother Oncol 2010; 96: 161-165.

20. Dimopoulos JCA, Lang S, Kirisits C et al. Dose-Volume Histogram Parameters and Local Tumor Control in Magnetic Resonance Image-Guided Cervical Cancer Brachytherapy. Int J Radiat Oncol Biol Phys 2009; 75: 56-63. 